\title{
Integrating ecosystem engineering and food webs
}

\author{
Dirk Sanders, Clive G. Jones, Elisa Thébault, Tjeerd J. Bouma, Tjisse van der Heide, \\ Jim van Belzen and Sébastien Barot
}

D. Sanders (d.sanders@exeter.ac.uk), Centre for Ecology and Conservation, College of Life and Environmental Sciences, Univ. of Exeter, Cornwall Campus, Penryn, TR10 9EZ, UK. - C. G. Jones, Cary Inst. of Ecosystem Studies, PO Box AB, Millbrook, NY 12545, USA. - E. Thébault, Inst. of Ecology and Environmental Sciences iEES (CNRS, UMPC, IRD, INRA), Univ. Pierre et Marie Curie, Batiment A, 7 quai St Bernard, FR-75252 Paris Cedex 05, France. - S. Barot, Inst. of Ecology and Environmental Science-Paris (CNRS, UMPC, IRD, INRA), Ecole Normale Supérieure, 46 Rue d'Ulm, FR-75230 Paris Cedex 05, France. - T. J. Bouma and J. van Belzen, Dept of Spatial Ecology, Royal Netherlands Inst. for Sea Research, PO Box 140, NL-4400 AC Yerseke, the Netherlands. - T. van der Heide, Dept. od Aquatic Ecology and Environmental Biology, Inst. for Water and Wetland Research, Radboud Univ. Nijmegen, Heyendaalsweg 135, NL-6525 AJ Nijmegen, the Netherlands, and: Centre for Ecological and Evolutionary Studies (CEES), Univ. of Groningen, PO Box 11103 , NL-9700 CC Groningen, the Netherlands.

\begin{abstract}
Ecosystem engineering, the physical modification of the environment by organisms, is a common and often influential process whose significance to food web structure and dynamics is largely unknown. In the light of recent calls to expand food web studies to include non-trophic interactions, we explore how we might best integrate ecosystem engineering and food webs. We provide rationales justifying their integration and present a provisional framework identifying how ecosystem engineering can affect the nodes and links of food webs and overall organization; how trophic interactions with the engineer can affect the engineering; and how feedbacks between engineering and trophic interactions can affect food web structure and dynamics. We use a simple integrative food chain model to illustrate how feedbacks between the engineer and the food web can alter 1) engineering effects on food web dynamics, and 2) food web responses to extrinsic environmental perturbations. We identify four general challenges to integration that we argue can readily be met, and call for studies that can achieve this integration and help pave the way to a more general understanding of interaction webs in nature.
\end{abstract}

All species are affected by their physical environment. Because ecosystem engineering species modify the physical environment and belong to food webs, such species are potentially one of the most important bridges $\frac{n}{2}$ between the trophic and non-trophic. We examine how to integrate the so far, largely independent research areas of ecosystem engineering and food webs. We present a conceptual framework for understanding how के engineering can affect food webs and vice versa, and how feedbacks between the two alter ecosystem dynamics. With appropriate empirical studies and models, integration is achievable, paving the way to a more general understanding of interaction webs in nature.

\section{Why integrate ecosystem engineering and food webs?}

The study of food webs has long been central to basic and applied understanding of stability and change in community organization and ecosystem functioning (Pimm 1982, Montoya et al. 2006, Mougi and Kondoh 2012). Recently, however, there have been calls to broaden the scope of food web studies to incorporate non-trophic interactions (Olff et al. 2009, Bascompte 2010, Kéfi et al. 2012); i.e. move toward the study of interaction webs that may better reflect reality (Ings et al. 2009). Ecosystem engineering, the physical modification of the environment by organisms (Jones et al. 1994, 1997), may well be the most common and influential kind of non-trophic interaction. Research over the last two decades has revealed that ecosystem engineering is nearly as ubiquitous as trophic interactions (Wright and Jones 2006, Hastings et al. 2007, Jones and Gutierrez 2007), and has the potential to alter the architecture and dynamics of entire interaction webs (Olff et al. 2009, Golubski and Abrams 2011, Kéfi et al. 2012) and many ecosystem processes (Dangerfield et al. 1998, Gutiérrez and Jones 2006, Lavelle et al. 2006). Because species are affected by their physical environment, and because ecosystem engineers are food web members and modify the physical environment, their dual role is potentially one of the most important bridges between the trophic and non-trophic.

Current food web models are parameterized on the basis of trophic connections. Any engineering effects on 
nodes and links are subsumed and represented as trophic influences. The engineering is therefore implicitly included but not explicitly parameterized, and this therefore precludes assessment of the relative influences of trophic interactions and engineering. Explicit exposure is needed if the engineering is a significant component and we seek to understand altered dynamics under externally-driven changes in species abundances or composition; or if there are changes in physical environmental factors modified by the engineer (e.g. thermal buffering, wave attenuation); or if the engineer is sensitive to those changes and modifies other physical environmental factors. Explanations or predictions based solely on trophic considerations could fail because the engineered environment will change if engineer density and per capita engineering activity changes. Although such considerations are very relevant to understanding changes in food webs in response to human-induced environmental change, e.g. climate or nutrient enrichment (Micheli 1999, Brown et al. 2010), the degree to which ecosystem engineers in food webs can mitigate or amplify extrinsic perturbations is largely unknown.

More explicit exposure of ecosystem engineering has applied relevance. Two examples illustrate the point. First, management practices in the UK were failing to stem widespread decline of the endangered large blue butterfly Maculinea arion whose larvae parasitize the ant Myrmica sabuleti. The ants require a warm environment to thrive. Due to myxomatosis, there were widespread declines in rabbit populations. This led to decreased grazing, increased turf height and, via shading, cooling of the ant environment, loss of the ant, and replacement by ant species unsuitable for $M$. arion. The decline was reversed when management practices were shifted from herbivore exclusion to a focus on grazing as a means of regulating turf height, hence environmental temperature (Thomas et al. 2009). Second, kelp forests create clear calm habitats for diverse near-shore communities via engineering effects on current flow and velocity, sediment deposition and sediment stabilization (Dayton 1985). Kelp forest persistence depends on managing offshore human fishing. Fishing reduces pinniped prey for killer whales that then switch to near-shore predation on sea otters, reducing otter predation on sea urchins that then consume kelp, converting kelp forests to more turbulent, turbid sea urchin barrens (Estes and Palmisano 1974, Estes et al. 1998).

If the study of food webs is to move toward the study of interaction webs for both theoretical and practical reasons, then merging ecosystem engineering dynamics (Cuddington et al. 2009, Jones et al. 2010, Jones 2012, Raynaud et al. 2013) with food web structure and dynamics seems a logical place to start. We begin by identifying how ecosystem engineering can affect the nodes and links of food webs and overall organization; how trophic interactions with the engineer can affect the engineering; and how feedbacks between engineering and trophic interactions can affect food web structure and dynamics. We then use a simple tri-trophic food chain model to illustrate the importance of feedbacks between the engineering effects and trophic interactions with respect to 1 ) the dynamics and stability of the food chain and 2) the response of the food chain to extrinsic environmental perturbation. Finally we identify
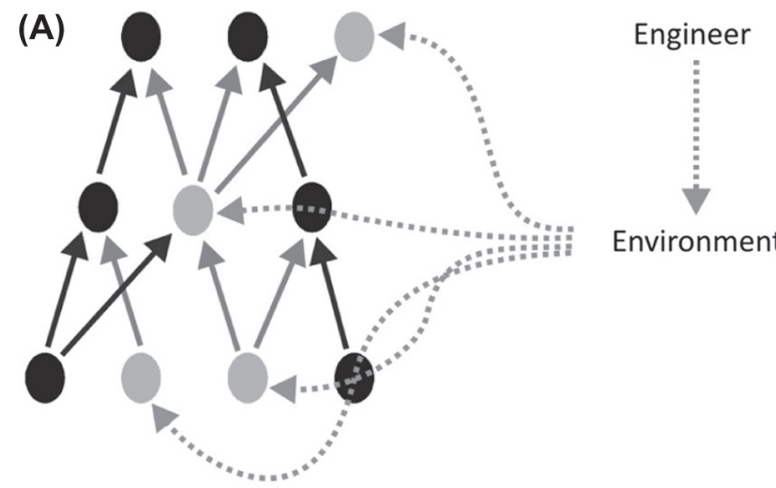

Environment
(B)

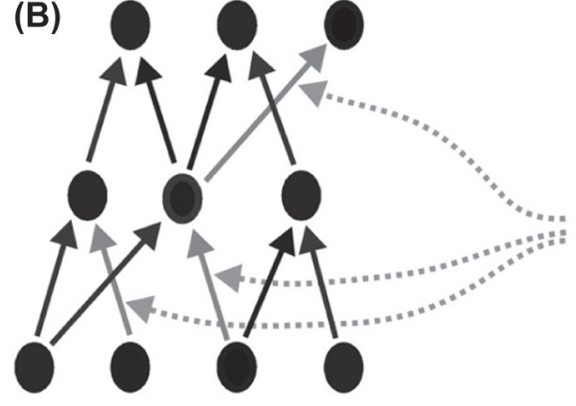

Engineer

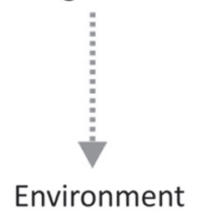

Figure 1. Impact of ecosystem engineering on the structure of food webs via (A) node modulation, and (B) link modulation. Nodes and links in grey colour indicate parts of the food web altered by ecosystem engineering. Node modulation also includes a subsequent change in links from these nodes to the rest of the food web.

four general challenges to integration that we think can readily be met, and call for studies that can achieve such integration and help pave the way to a more general understanding of interaction webs in nature.

\section{How ecosystem engineering can shape food webs}

\section{Node and link modulation}

In webs of ecological interactions, species can be viewed as network nodes linked by trophic and non-trophic interactions (Solé and Montoya 2001). By changing the environmental context, ecosystem engineers can affect the nodes, i.e. the number of species that are present and their densities, and the number and strength of links among these species (Fig. 1). We refer to these as node and link modulation, respectively. Engineering modulates the niche space for species. Abiotic factors influenced by the engineering act as environmental filters (Mayfield et al. 2009) allowing or preventing species with certain traits to enter the community (Shachak et al. 2008). Node modulation also results in link modulation (e.g. new links with new species, or lost/reconfigured links with excluded species), with the potential for cascading effects throughout the web. Increased abiotic resource availability due to engineering can result in the addition of new producer species or altered producer biomass, which may then propagate to higher trophic levels. For example, in the Negev Desert, Israel, shrub interception and deposition of dust and detritus at the base results in a loose, un-crusted soil mound. The mounds locally increase infiltration of run-off water resulting in 'islands of fertility' with a diversity and abundance of 
plant species not found in adjacent, un-mounded habitat types (Wright et al. 2006). Direct link modulation is also possible. For example, a decrease in the feeding rate of insect herbivores caused by shading and concomitant lower temperature leads to a reduction in the degree to which top-down control can affect plant biomass (Chase 1996).

\section{Abiotic conditions and resources}

Engineer node and link modulation can be thought of as operating on three, non-exclusive pathways: altered abiotic conditions, consumable abiotic resources, and non-trophic abiotic resources (for explanation of how engineers cause such changes see Jones et al. 2010).

1) Abiotic conditions are non-resource abiotic factors that affect species, such as temperature, $\mathrm{pH}$, redox, wind and sediment deposition. Conditions are commonly altered by engineers (Jones et al. 2010). For example, cushion plants in the Andes buffer temperature extremes and reduce evaporation of soil moisture, resulting in altered species composition and abundances for plants living in cushions compared to adjacent areas (Badano et al. 2006); i.e. node modulation of network structure. Similarly, Spartina attenuation of water velocity and substrate stabilization changes the structure of beach communities (Bruno 2000). Woodward et al. (2010) report that even modest temperature increases in stream ecosystems can have large effects across multiple levels of community organization including changes in the mean body size of top predators, turnover in community composition, and lengthening of food chains. This indicates a substantial potential for both link and node modulation via engineer-induced changes in abiotic conditions; in this latter example, tree shading of a stream reducing water temperature.

2) Consumable abiotic resources. Engineering that locally concentrates or dilutes abiotic resources is also common (Jones et al. 2010). Examples include the trapping and storage of runoff water (Wright et al. 2006) and nutrient leaching via soil macropore formation (Knight et al. 1992). Many ecosystem engineers increase decomposition rates of litter via physical fragmentation and physical incorporation into soil and sediments. Examples include earthworms (Parmelee et al. 1998), termites (Holt and Lepage 2000), crabs (Gutiérrez et al. 2006), and marine sediment infauna (Hansen and Kristensen 1997). Such species often concentrate or disperse organic matter in patches at different spatial scales, via, for example, the burrows they dig (Gutiérrez et al. 2006), their foraging activities (Brody et al. 2010), or the particulate material they trap with physical structures (Bos et al. 2007). The resulting change in resource distributions can lead to a net increase or decrease in the availability of mineral nutrients and a long-term increase or decrease in primary production (Barot et al. 2007) via node modulation at the base of the food web. Recent work (Rooney et al. 2006) indicates that strong asymmetries in productivity and turnover rates between energy channels in food webs increase stability. Because many engineers can affect nutrient flows and primary productivity, they may well be a major cause of asymmetry, and hence food web stability.
3) Non-trophic resources are various kinds of autogenicallyand allogenically-engineered (Jones et al. 1994) space, such as living-, enemy-free- or enemy-rich-, competitorfree-, and mutualist-rich-space (Jones et al. 2010). Common examples of link modulation via engineered space are prey refuges and predator-concealing cover created by plant architecture, leaf litter, burrows, shells, and dead wood (Gutiérrez and Iribarne 1999, Pintor and Soluk 2006); even the shade caused by phytoplankton populations (Radke and Gaupisch 2005). In general, engineered space that decreases predation should reduce trophic interaction strengths in food webs. In contrast, engineered space that increases predator attack rates (Hopcraft et al. 2005) would be expected to increase trophic interaction strengths, as illustrated by certain plant species that provide optimal structure for spider webs (Pearson 2010).

There is often overlap among the three pathways with respect to the responding organisms. Light can be a resource for one species (e.g. a sapling competitor) and a condition for another (e.g. understory species intolerant of high light). For a given species, an abiotic factor can be a resource at one value and a condition at another (e.g. water as a resource vs flooding stress). Many engineers affect communities via more than one pathway. For example, trees provide habitat structure, a non-trophic resource; change abiotic conditions such as temperature and humidity; and reduce light availability as a consumable resource for other plants.

Irrespective of overlap, consideration of pathways helps generate expectations as to engineer influence on nodes and links. The more precisely we can describe how the engineering operates the better we might be able to predict the trophic impact. The three pathways operate in different ways and are not equally sensitive to a given environmental change. For example, if a tree is considered from the perspective of its influence on temperature, then we need to consider its capacity to buffer temperature change, and the direct effect of temperature on the tree, and how this then affects thermal buffering capacity. In contrast, if the tree is considered solely as a predator refuge (ignoring thermal effects on predator and prey since we are focussing here on only on engineered space), we only need to consider whether temperature change might alter the structural refuge. In general, we think it useful to consider the engineering pathways relative to what is changing in the environment due to external forcing, and then consider the capacity of the engineer to attenuate or amplify such forcing and the sensitivity of the engineer to that forcing.

\section{The part of the food web influenced by engineering}

Engineering pathways can influence the whole food web, a certain trophic level or compartment, or one or more species within or across levels in the food web (Fig. 2). The provision of a specific kind of non-trophic resource, like a burrow, might be expected to have a more speciesspecific impact (Fig. 2A), such as crayfish burrows used by larvae of a dragonfly species (Pintor and Soluk 2006). The change in a resource, such as light via shading, is more likely to affect many species within the engineered environment at the same trophic level (e.g. photoautotrophs; Fig. 2B). Engineering may affect a subset of species across trophic levels (Fig. 2C; e.g. mound building by 


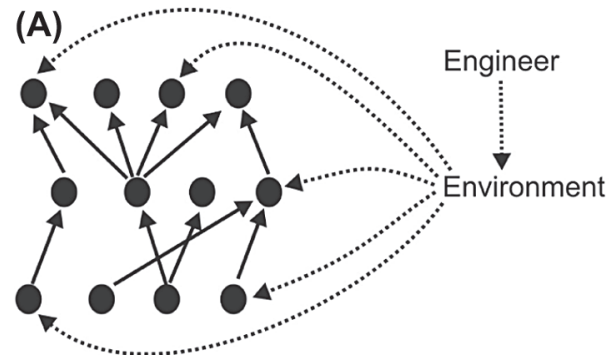

(B)
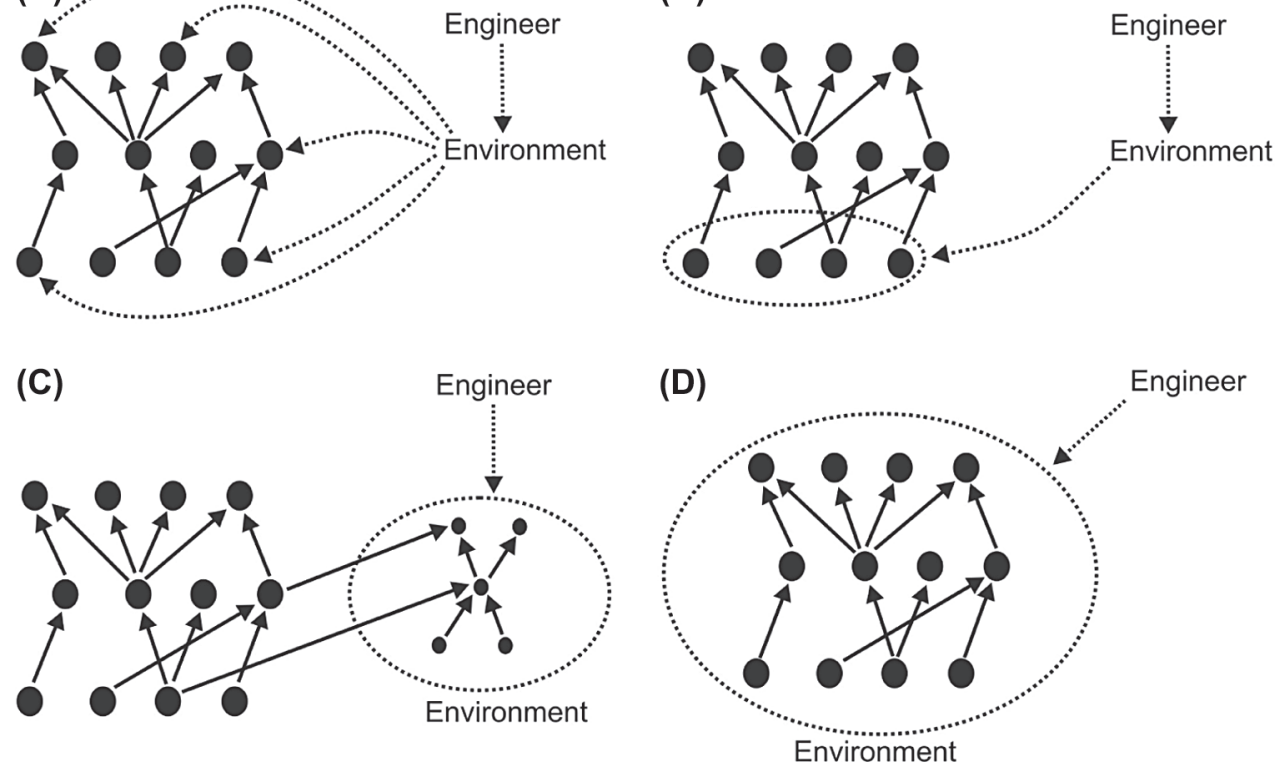

Figure 2. Ecosystem engineering can affect: (A) a sub-set of species at different trophic levels; (B) one trophic level; (C) a food web compartment; or (D) all species in a food web by, for example, creating the entire environment in which the food web occurs.

ants, Sanders and van Veen 2011). This subset could encompass a substantial fraction of the web if the habitat modification involves many engineering pathways affecting most features of an ecosystem, such as zebra mussels (Strayer et al. 1999) or North American beaver (Anderson and Rosemond 2010). The de novo creation of an entirely new kind of habitat by an ecosystem engineer such as water filled bromeliads and tree holes (Kitching 1971) or coral reefs (Fig. 2D) illustrates the situation where the engineer has a 'global' impact on the food web by creating the elemental structure. It is important to note, however, that because these kinds of food webs are unlikely to be completely isolated, it might be necessary to take the engineer and its activity into account in a larger scale food web. For example, predators of tree-hole mosquitoes do not invariably live in tree holes (Medlock and Snow 2008), and juveniles of many coral reef fish species migrate between corals and nearby mangroves or seagrass meadows to feed and be fed upon (Nagelkerken et al. 2000).

A beaver does not belong to the aquatic food web in the pond it creates (other than as a likely minor extrinsic source of nutrient input via urine and feces). Because there are no trophic links back to beaver, the pond can be treated as a 'found' abiotic environment with respect to food web structure and dynamics; albeit an environment that reflects, in part, whatever drives engineer dynamics, including its trophic interactions elsewhere. In contrast, many of the species that depend on the shelter from wave action provided by coral reefs, also feed on corals or feed on coral-consuming species (Tribollet and Golubic 2011). When engineers are trophically coupled to food webs as producers, consumers or decomposers, there is obviously the potential for trophic feedbacks to alter engineer density and engineering activity. This can then lead to a change in the degree to which the environment is modified, subsequent modification of trophic interactions, and so on.

\section{Trophic and engineering control, outcomes and feedbacks}

\section{Engineer net impact and feedbacks}

The net effect of an engineer on a food web in which it is embedded will be the result of three component effects (Fig. 3). 1) Engineering effects. These will depend on the number and positions of affected nodes and links, and the directions and magnitudes of these effects that result from the three kinds of engineering pathways. 2) Trophic effects of the engineer. These will depend on trophic position, trophic connectivity (number of links) between the engineer and other species, and quantitative per capita contribution to energy and nutrient flows. 3) Positive or negative trophic feedbacks to the engineer (Jones et al. 2010) via their predators, prey, mutualists and competitors.

It is possible to distinguish engineering effects from trophic effects of the engineer. For example, Prugh and Brashares (2012) studied the impact of kangaroo rats on a grassland community. Engineering activity (i.e. burrow construction) was a primary influence on plant and small mammal community organization; whereas trophic effects (i.e. herbivory and seed predation) were a primary influence on invertebrate community organization and increased lizard abundances. Both engineering and trophic effects were directionally aligned, and both resulted in facilitation of other species. In contrast, a study by Sanders and van Veen (2011) indicated that there were two opposing influences of ants on a grassland food web. There was a bottom-up engineering effect resulting from increased primary productivity via mound-building by ants (i.e. abiotic conditions and/or consumable abiotic resource pathways) leading to an increase in decomposer, herbivore and parasitoid density; and a top-down, trophic effect of ant predation counteracting the bottom-up effect on herbivores and parasitoids. The study further demonstrated that the 

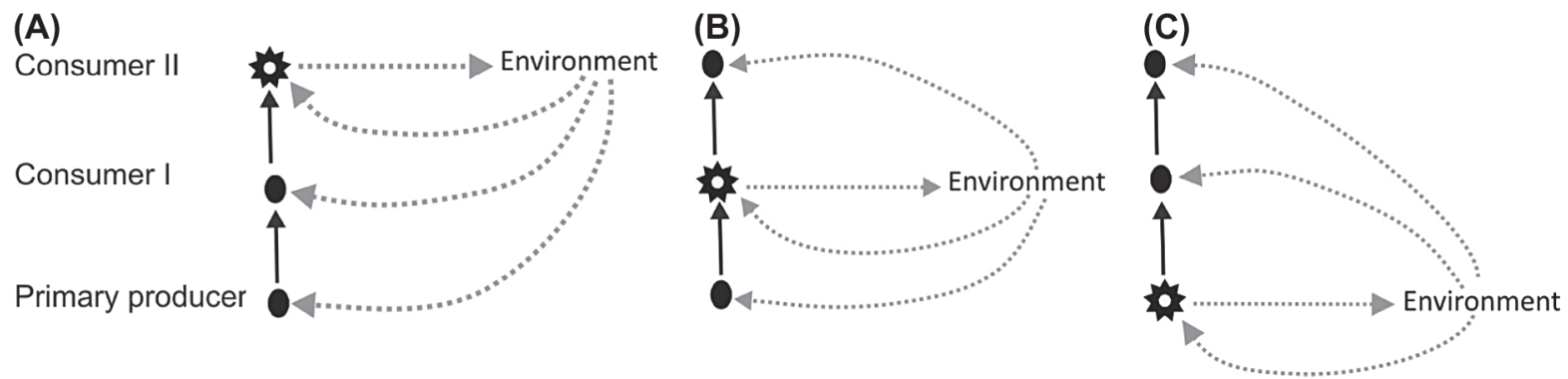

Figure 3. An ecosystem engineer (denoted by the star) can create feedbacks to engineer density and per capita engineering activity via trophic effects (black arrows illustrating for example, predator and engineer prey) and engineering effects (dotted arrows via environment; here shown as affecting all food web members, although this does not have to be the case). The trophic position of the engineer can also vary: (A) top; (B) intermediate; (C) base.

relative strength of engineering to trophic effects, and the resulting positive or negative net impacts, depended on engineer density. Assuming that engineering and trophic effects can vary in magnitude and direction as illustrated by the above two examples, we can imagine that given equal strength and opposite directions, trophic and engineering effects on the same set of species could nullify each other such that underlying joint influences of the engineer would not be detectable unless teased apart. Furthermore, while it is clear that engineering and trophic effects of engineers may influence the same species positively or negatively (Pintor and Soluk 2006, Sanders and van Veen 2011), they can also affect different species (Prugh and Brashares 2012).

Ecosystem engineers can obviously belong to different trophic levels (Fig. 3). For example, many ants (Sanders and van Veen 2011) and crayfish (Pintor and Soluk 2006) are predators; leaf cutter ants (Jonkman 1980) and many reef-building molluscs (Gutiérrez et al. 2003) are at intermediate trophic positions; and plants (Badano et al. 2006), including diatoms (Daborn et al. 1993), are at the base of food webs. The trophic position of the engineer may well be important. If the engineer is at the base of the food web, engineer density, hence engineering effect magnitudes, can be influenced by higher trophic levels. In the absence of strong top-down control by predators (Pace et al. 1999) herbivores may not only affect plant biomass, but could also affect biomass-dependent engineering effects (e.g. refugia). For example, sandy sediments in the Bay of Fundy are stabilized by mucopolysaccharide secretions of diatoms, which are consumed by amphipods, leading to destabilized sediments at high amphipod densities. High densities of migratory sandpipers that feed on amphipods can cause re-stabilization of sediments (Daborn et al. 1993). The system illustrates a coupled trophic-engineering cascade (Jones et al. 1997) where the degree of environmental control by the engineer at the base of the food web depends on relative densities of higher trophic levels. A similar situation occurs with kelp forests and the pelagic food web mentioned earlier (Estes and Palmisano 1974, Estes et al. 1998). These kinds of influences can also apply to decomposer food webs (Schmitz 2010), such as the effects of earthworm predators on earthworm density and engineering effect magnitudes.

The combination of trophic and engineering interactions in food webs may result in: 1) trophic feedbacks to engineers; and 2) engineering feedbacks to consumers.
Trophic feedbacks to the engineer (Jones et al. 1997) can occur via prey, predators, mutualists or competitors of the engineer (Fig. 3), although we will illustrate this only for predators and prey. Feedbacks will depend on 1) the direction (positive or negative) and strength of the engineering effects; 2) the trophic position of the engineer; and 3) the trophic level(s) affected by the engineering. We should, for example, observe a strong positive feedback to the engineer when engineering activity increases the abundance of engineer resources while decreasing the abundance of engineer predators. Conversely, we can expect strong negative feedbacks when engineering activity decreases the abundance of engineer prey and increases the abundance of engineer predators. When the engineer is an apical predator (as in Sanders and van Veen 2011), a net positive feedback to the engineer therefore requires positive engineering effects on prey abundance to exceed negative effects on prey abundance due to engineer consumption of prey. The net outcome of engineering at intermediate trophic levels will likely depend on the relative strength of bottom-up and top-down feedbacks. For example, reef construction by molluscs (Gutiérrez et al. 2003) can result in positive trophic feedbacks via hydrodynamic influences on food supply (i.e. consumable abiotic resource pathway), and negative trophic feedbacks via creation of refugia for mollusc predators (i.e. non-trophic resource pathway via an increase in engineered enemy-rich space) such as increased rates of consumption of the oyster Crassostrea virginica by the mud crab Panopeus herbstii (Grabowski and Powers 2004). Such kinds of negative trophic feedbacks via engineer-created predator refuges (i.e. non-trophic resource pathway) may be common. Holt and Barfield (2013) modelled the effects of increased vegetation biomass (or a correlated structural feature) on predation success. The model illustrates the rich variety of net effects that can occur in simple community models when the potential diversity of feedbacks is taken into account. A predator that initiates a trophic cascade that increases plant biomass and habitat structure may experience positive or negative feedbacks depending on whether or not this creates more prey refuges or increased predation success.

The above indicates a large potential for feedbacks between trophic and engineering interactions to alter food web dynamics. Nevertheless, the degree to which the combination of engineering and tropic interactions does lead to new dynamics and equilibria is largely unknown. We do not 
know when outcomes will be the result of engineering and trophic relationships operating independently $v s$. interactively, and we do not know how the inclusion of engineering might alter food web responses to extrinsic perturbation.

\section{Engineer-food web dynamics in a simple food chain}

To shed some light on the above questions, we modified a well-studied three-species food chain model to explore how engineering pathways (i.e. abiotic conditions, consumable abiotic resources, non-trophic resources), per capita engineering activity, the trophic level(s) of engineering effects, and the trophic position of the engineer might be expected to affect food chain structure and dynamics in the absence and presence of extrinsic environmental perturbation. In all situations, we compared food chain structure and dynamics to a 'control' situation in which the engineer had the same kind and magnitude of effects on the food chain, but was extrinsic to the food chain (i.e. no trophic feedback to the engineer).

\section{Modelling methods}

The underlying model has a primary producer and two levels of consumers, i.e. an herbivore and a predator (Rosenzweig 1973, McCann and Yodzis 1995), and has been used, for example, to assess the impact of omnivory (McCann et al. 1998a) and predator-prey body mass ratios (Otto et al. 2007) on food chain stability. The dynamics of species are described by the following system of equations:

$$
\left\{\begin{array}{l}
\frac{d R}{d t}=R\left(1-\frac{R}{K}\right)-\chi_{C} y_{C} \frac{C R}{R+R_{0}} \\
\frac{d C}{d t}=-\chi_{C} C+\chi_{C} y_{C} \frac{C R}{R+R_{0}}-\chi_{P} y_{P} \frac{P C}{C+C_{0}} \\
\frac{d P}{d t}=-\chi_{P} P+\chi_{P} y_{P} \frac{P C}{C+C_{0}}
\end{array}\right.
$$

where $R$ is the resource density; $C$ is the intermediate consumer density; $P$ is the top predator density; $K$ is the resource carrying capacity; $R_{0}$ and $C_{0}$ are the half saturation densities of the consumer and predator functional responses for the resource and intermediate consumer, respectively; $x_{i}$ is the mass-specific metabolic rate of species $i$; and $y_{i}$ is the ingestion rate per unit metabolic rate of species $i$. Values of parameters were defined according to McCann and Hastings (1997) for the case of a system with a stable equilibrium in the absence of any ecosystem engineering effects (Table 1).

The exact shape of engineer effects on species parameters in such a model is unknown (Kefi et al. 2012). We thus followed the suggestion of Kefi et al. (2012) for incorporating non-trophic interactions into food webs by expressing engineer effects on species parameters in the model as a saturating function of engineer density. We considered an ecosystem engineer that could either affect consumer ingestion rates (Eq. 2) or resource carrying capacity (Eq. 3):

$$
\begin{aligned}
& y_{i}=\frac{y_{M i}\left(\alpha E+N_{0}\right)}{E+N_{0}} \\
& K=\frac{K_{M}\left(\alpha E+N_{0}\right)}{E+N_{0}}
\end{aligned}
$$

where $y_{M i}$ and $K_{M}$ are respectively the consumer ingestion rate and the resource carrying capacity in the absence of engineering; $E$ is the density of the ecosystem engineer; and $\alpha$ corresponds to the direction and strength of the per capita engineering effect (Jones et al. 1994). According to Eq. 2 and 3, consumer ingestion rates and resource carrying capacity are respectively $y_{M i}$ and $K_{M}$ when there is no engineer, and consumer ingestion rates and resource carrying capacity saturate at the values $\alpha y_{M i}$ and $\alpha K_{M}$ when the engineer is abundant. If $\alpha<1$, the engineer decreases consumer ingestion rates or decreases resource carrying capacity; if $\alpha>1$ it increases the ingestion rates or increases the carrying capacity; and if $\alpha=1$, the engineer has no effect $\left(y_{i}=y_{M i}\right.$ and $\left.K=K_{M}\right)$. Parameter values for this aspect of the model are also shown in Table 1.

The above can be considered to represent two general kinds of situations: 1) the engineer affects food chain links (i.e. increases or decreases ingestion rates) via pathways that alter abiotic conditions (e.g. thermal buffering or exposure) and/or non-trophic resources (i.e. engineered structure per se; e.g. prey refuges or predator ambush locations); or 2 ) the engineer increases or decreases consumable abiotic resources (i.e. the third pathway via effects on resource carrying capacity $K_{M}$ ).

To address how engineering might alter food chain responses to extrinsic perturbation, we simulated the effects of nutrient enrichment by increasing the value of the background resource carrying capacity in absence of engineer $\left(K_{M}\right)$

Table 1. Parameter values used in model simulations. Letters refer to simulation results presented in panels of Fig. 4.

\begin{tabular}{lll}
\hline Symbol & \multicolumn{1}{c}{ Definition } & \multicolumn{1}{c}{ Values } \\
\hline$K_{M}$ & resource carrying capacity & $(\mathrm{A}),(\mathrm{C}),(\mathrm{E}): 1(\mathrm{~B}),(\mathrm{D}),(\mathrm{F}):[1,1.4]$ \\
$x_{C}$ & mass-specific metabolic rate of the intermediate consumer & 0.4 \\
$y_{M C}$ & ingestion rate per unit metabolic rate of the intermediate consumer & 2.009 \\
$R_{O}$ & half saturation density of the resource & 0.5 \\
$x_{P}$ & mass-specific metabolic rate of the top predator & 0.01 \\
$y_{M P}$ & ingestion rate per unit metabolic rate of the top predator & 5 \\
$C_{O}$ & half saturation density of the intermediate consumer & 0.9 \\
$N_{O}$ & half saturation density of the engineering effect & 1.5 \\
$\alpha$ & direction and strength of the per capita engineering effect & (A), (C), (E): [0,2] (B), (D): $0.5 ;(\mathrm{F}): 1.2$ \\
$E$ (if external engineer) & density of the ecosystem engineer & (A) $2.467,(\mathrm{~B}) 0.226,(\mathrm{C})$ and (D) 0.863 \\
\hline
\end{tabular}


for situations where the engineer either decreases consumer ingestion rates $(\alpha=0.5)$ or increases resource carrying capacity $(\alpha=1.2)$. We chose nutrient enrichment as a food chain perturbation because it is obviously relevant - human-induced nitrogen and phosphorus enrichment of ecosystems is common (Vitousek et al. 1997, Tilman et al. 2001) - and because it has also received substantial theoretical attention (Hulot et al. 2000, Cross et al. 2006), albeit without explicit consideration of how engineering might affect outcomes.

We analyzed species densities and dynamics at steady state by constructing bifurcation diagrams that allowed comparison of engineering effects when the engineer was trophically embedded versus extrinsic to the chain at varying levels of $\alpha$ or $K_{M}$ in six illustrative scenarios (of many possible); three without and three with extrinsic environmental perturbation. Each scenario consisted of simulations of species dynamics over 10000 time steps for 40 varying values of $\alpha$ or $K_{M}$. In each simulation, we recorded the local minima and maxima of resource, consumer and predator densities during the last 3000 time steps, thereby avoiding transient dynamics. When the system reached a stable fixed point for a given $\alpha$ or $K_{M}$ level, we obtained a single value for resource, consumer and predator densities (i.e. at equilibrium, the minimum and maximum densities for a species are identical). When the system showed either cyclic or chaotic dynamics, minimum and maximum densities for a species differed and multiple local minima and maxima species density values could occur although in most simulations here we found only one minimum value and one maximum value for species density corresponding to a single-period cycle.

We first examined three scenarios without extrinsic perturbation (summarized in Table 2) to investigate the consequences of the direction and strength of the per capita engineering effect (i.e. $\alpha$ effects) on species dynamics. We then examined three otherwise comparable, but more limited scenarios, where extrinsic perturbations affected abiotic resources at the base of the food web (i.e. extrinsic $K_{M}$ effects, Table 2). We simulated this by increasing the value of the resource carrying capacity in absence of engineer $\left(K_{M}\right)$.
For all scenarios, as noted briefly above, we evaluated the effects of engineer integration into the food chain by defining an otherwise comparable 'control' scenario where we excluded feedbacks by making the engineer external to the food chain at an invariant density equal to the density of a trophically-embedded engineer when $\alpha=1$, but with otherwise identical parameterization (i.e. the engineer affects the food chain exactly the same way but is not one of the three species in the food chain). Comparing the two kinds of situations in all scenarios allowed us to assess how feedbacks might depend on engineering pathway, engineer trophic position, trophic position of the engineering effect, and extrinsic environmental perturbation.

\section{Model results}

Scenarios 1-3 (Fig. 4A, C, E) show how the strength and direction of the per capita engineering effect (i.e. varying levels of $\alpha$ ) on abiotic conditions and/or non-trophic resources (Fig. 4A, C), and the capacity of the engineer to alter consumable resources (Fig. 4E) can modify food chain structure and dynamics. The strength and direction of the per capita engineering effect modified species densities and dynamic behaviour depending on engineering pathway, the trophic level(s) of engineering effects, and the trophic position of the engineer. When engineering activity increased intermediate and/or top consumer ingestion rates or resource carrying capacity $(\alpha>1)$, it always led to cyclic dynamics. While this result is perhaps not surprising increased trophic interaction strengths and increased productivity are known to be destabilising in such models (Rosenzweig 1971, McCann et al. 1998a) - the degree of destabilization was markedly modified by feedbacks between the engineer and the food chain. When the engineer affected ingestion rates and was also the intermediate consumer (scenario 1, Fig. 4A), an increase in per capita engineering effects led to cyclic dynamics of larger amplitude than when the engineer was at a similar initial density but external to the food chain; feedbacks resulting from the trophic position of the engineer were destabilizing. In contrast, when the

Table 2. Description of the six different scenarios investigated in the model. In scenarios 1, 2, 4 and 5, engineer effects were assumed to have the same direction and magnitude of per capita engineering effect, set by $\alpha$. Scenario 1 could, for example, correspond to a situation where an herbivore affects abiotic conditions that increase $(\alpha>1)$ or decrease $(\alpha<1)$ its own ingestion rate as well as that of the top predator (e.g. increases local temperature via removal of over-story vegetation leading to increased understory growth, while at the same time increasing predator detection due to loss of cover). Scenario 2 could, for example, correspond to situations where a primary producer increases or decreases temperature depending on cover and albedo, such that both herbivore and predator ingestion rates increase or decrease (Chase 1996). Scenario 3 could, for example, correspond to a situation where an herbivore increases or decreases local mineral nutrient retention and the rate of nutrient cycling via effects on soil structure (e.g. burrowing herbivores). Scenarios 4, 5 and 6 address selected subsets of scenarios 1-3 under situations in which externally forced abiotic resources increase.

\begin{tabular}{|c|c|c|c|}
\hline Scenario & Engineer trophic position & Effects of the engineer & Parameters evaluated \\
\hline 1 & intermediate consumer & $\begin{array}{l}\text { modifies ingestion rates of intermediate consumer } \\
\text { and top predator }\end{array}$ & $\begin{array}{l}\text { effects of direction and magnitude of per } \\
\text { capita engineering effects, i.e. varying } \\
\alpha \text { levels }\end{array}$ \\
\hline 2 & primary producer & $\begin{array}{l}\text { modifies ingestion rates of intermediate consumer } \\
\text { and top predator }\end{array}$ & \\
\hline 3 & intermediate consumer & modifies carrying capacity of primary producer & \\
\hline 4 & intermediate consumer & $\begin{array}{l}\text { decreases ingestion rates of intermediate consumer } \\
\text { and top predator }(\alpha=0.5)\end{array}$ & $\begin{array}{l}\text { effects of an extrinsic perturbation, } \\
\text { simulated as an increase of carrying } \\
\text { capacity, i.e. increasing } K_{M} \text { levels }\end{array}$ \\
\hline 5 & primary producer & $\begin{array}{l}\text { decreases ingestion rates of intermediate consumer } \\
\text { and top predator }(\alpha=0.5)\end{array}$ & \\
\hline 6 & intermediate consumer & increases carrying capacity of primary producer $(\alpha=1.2)$ & \\
\hline
\end{tabular}



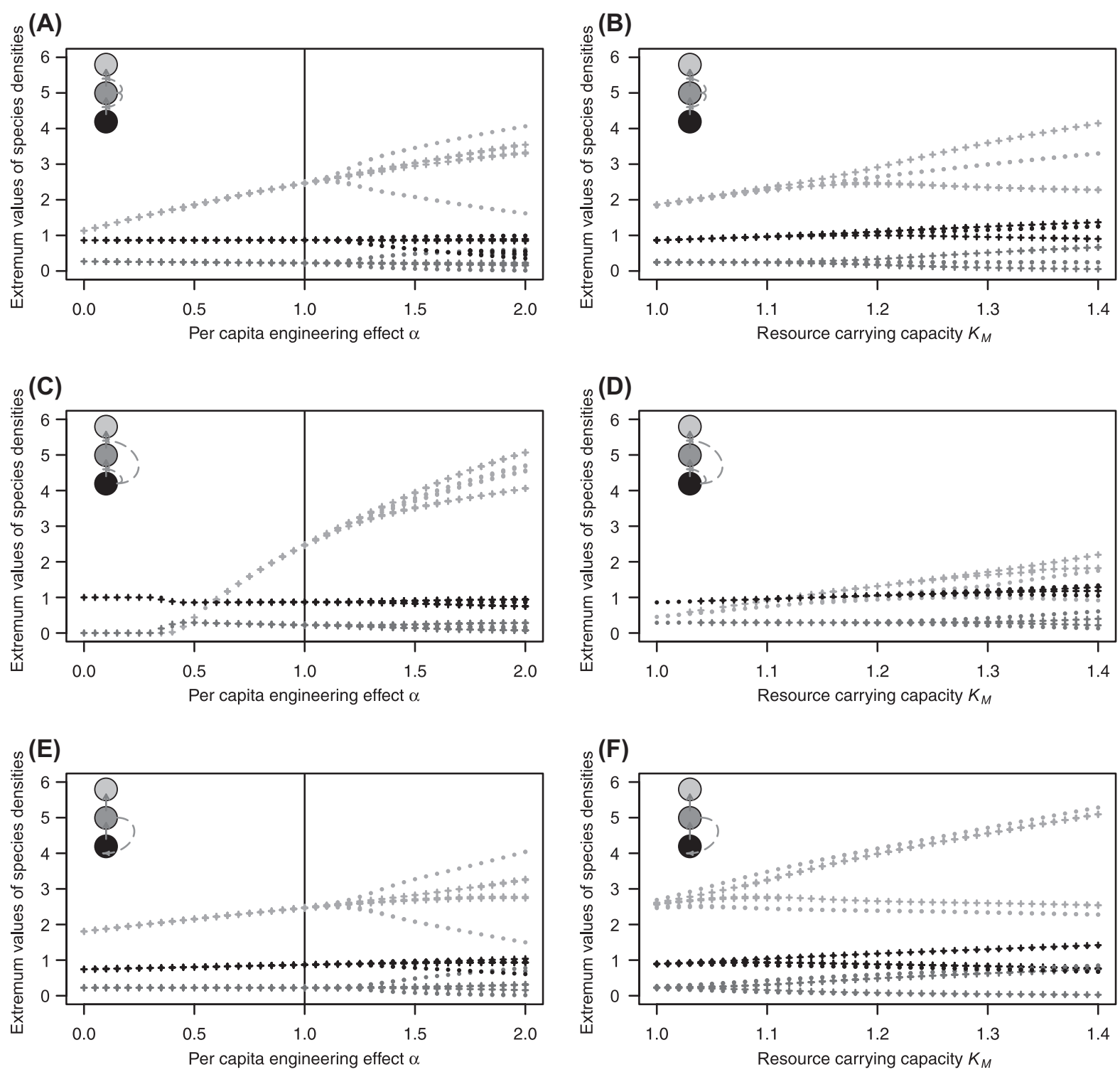

Figure 4. Bifurcation diagrams reporting local minima and maxima of species densities at steady state (extremum values) for six different ecosystem engineering scenarios in a three-species food-chain model as a function of the direction and strength of the per capita engineering effect, $\alpha$ (left panels); and as a function of resource carrying capacity, $K_{M}$, simulating one kind of extrinsic perturbation - abiotic nutrient enrichment affecting the base of the food web (right panels). The engineer is either outside of the food chain ('control', densities represented by crosses) or embedded in the food chain (densities represented as filled circles). Densities are in black for the primary producer at the base of the food chain, dark grey for the intermediate consumer, and light grey for the top predator. $\mathrm{A}$ and $\mathrm{B}$ : the engineer is the intermediate consumer and affects its own ingestion rate and that of the top predator $(\alpha$ varying, A; $\alpha=0.5$, i.e., engineer decreases ingestion rates, B). C and D: the engineer is the primary producer and affects ingestion rates at both higher trophic levels ( $\alpha$ varying, $C ; \alpha=0.5$, i.e. engineer decreases ingestion rates, D). $\mathrm{E}$ and $\mathrm{F}$ : the engineer is the intermediate consumer and affects consumable abiotic resources (i.e. carrying capacity) of the primary producer ( $\alpha$ varying, $\mathrm{E} ; \alpha=1.2$, i.e. engineer increases carrying capacity, F).

engineer was the primary producer, feedbacks between the engineer and the food chain stabilized species dynamics (scenario 2, Fig. 4C). When the engineer was the intermediate consumer and affected resource carrying capacity, increases in per capita engineering effects also led to cycles of larger amplitude than when the engineer was at a similar density but external to the food chain (scenario 3, Fig. 4E). In this case, a positive feedback due to the trophic position of the engineer might explain the observed cycle amplification - an increase in engineer density led to an increase in resources for the primary producer that then increased the population growth rate of the engineer. Model results for these three scenarios indicate that engineering effects on food web stability might strongly depend on the trophic position of the engineer and complex feedbacks between engineering and trophic effects.

Scenarios 4-6 (Fig. 4B, D, F) illustrate how the inclusion of engineering can alter the outcomes for food chain structure and dynamics in the presence of extrinsic environmental perturbation, hence the degree to which engineers might 
mitigate or amplify externally forced environmental change. Our analysis showed that the effects of enrichment (i.e. increasing $K_{M}$ levels) on species densities and dynamics also depended on the trophic position of the engineer (scenarios 4, 5; Fig. 4B, D). In most cases, enrichment led to cyclic dynamics - also a typical finding for the classical model that does not include engineering (Rosenzweig 1971, Moore et al. 1993). However, the strength of the destabilizing effect of enrichment depended strongly on the trophic position of the engineer. When the engineer was the intermediate consumer (scenario 4; Fig. 4B), feedbacks between the engineer and the food chain strongly dampened destabilizing effects such that enrichment did not result in cyclic dynamics. When the engineer was the primary producer (scenario 5; Fig. 4D), the destabilizing effects of enrichment were amplified (i.e. cycles of larger amplitude), and enrichment effects on top predator density were weaker in comparison to the situation in which the engineer was at a similar density but external to the food chain. This latter finding can be explained by feedbacks resulting from the trophic position of the engineer. Enrichment increased engineer density leading to an increase in engineering activity, which then decreased consumer ingestion rates $(\alpha=0.5)$, attenuating enrichment effects on top predator density. In contrast, positive feedbacks arising from engineer-induced increases in resource carrying capacity (scenario 6; Fig. 4F) resulted in increased intermediate consumer density that amplified the destabilising effects of extrinsic enrichment (scenario 6; Fig. 4F). Overall, these three latter scenarios indicate that consequences of environmental perturbations on food webs with engineers - in this case nutrient enrichment - might depend on a complex mix of engineering and trophic effects and complex feedbacks. Given the recognized risks to ecosystem functioning and biodiversity posed by humaninduced increases in nutrient loading occurring in many ecosystems (Vitousek et al. 1997), predicting how ecosystem engineers might alter expected food web dynamics is clearly central.

Although the model we used is the simplest kind of food web with a restricted set of parameters, across all six scenarios, explicitly embedding an ecosystem engineer into the food chain often led to marked differences in density and stability relative to a food chain where the engineer was external. Embedding engineers altered engineer dynamics and food web dynamics. The simple scenarios exemplify the potential impact of the trophic position of the engineer and the impact of the engineer on trophic links. It is clear that further modelling and empirical work will be required to ascertain whether the effects we have described are amplified or mitigated when the engineer is embedded within a larger and more complex food web and where the engineers may impact only a few trophic links or virtually all of them.

\section{Integrating engineering and food webs: where next?}

Our provisional framework and findings from the simple food chain model illustrate how the combination of ecosystem engineering and food web structure and dynamics are expected to lead to different expectations for outcomes compared to situations in which they are considered to occur independently. Our work suggests that there is considerable potential for advancing understanding of both engineer dynamics and food web dynamics areas via their integration. To accomplish such integration we need empirical data on both aspects in the same system and integrative models. The flexible modelling framework of Kéfi et al. (2012) appears well-suited to integration for different types of models. As illustrated by our simple model, engineer effects on abiotic conditions, consumable abiotic resources, and non-trophic resources can all be encompassed in the presence and absence of extrinsic environmental perturbations. While we do not expect the outcomes for the simple tritrophic model to explicitly hold in more complex food webs, the effects of additional complexity nevertheless appear amenable to study. It is clear that we need to study a number of systems if we seek generalizations, so we suggest four general ways to move forward. The first two are empirical, while the other two involve modelling. Of course, modelling efforts will benefit from empirical studies and vice versa.

First, we appear to know less about the effects of the abiotic environment per se on the structure and dynamics of food webs than we know about how engineers alter abiotic environments. If so, then lack of knowledge of the former limits our ability to integrate the latter. Comparative and manipulative studies of known food webs in different abiotic states could help bridge this knowledge gap. Studies by Woodward et al. (2010) on temperature effects, and Petchey et al. (2010; temperature and connectance) are illustrative of what can be learnt, but many more such kinds of studies on well-characterized food webs are needed.

Second, many studies on ecosystem engineering appear to have been conducted in systems in which the food web is only partially characterized or not characterized. Conversely, systems with the best characterized food webs (van Veen et al. 2008, Woodward et al. 2010, Boit et al. 2012) appear to be those where the engineering is understudied or unstudied. The two need to be comprehensively combined in the same system and any manipulations need to target both aspects. Studying the engineering in already well-characterized food webs may well be easier than characterizing food webs in systems where the engineering has been studied.

Third, the degree to which abiotic variables should be explicitly modelled remains to be seen. This will likely depend on the degree to which such effects create lags and non-linearities, and the occurrence of complex abiotic interactions. In the simplest situation (and as with our model) they could be simply collapsed into engineer density and activity. If, however, lags and non-linearities are influential and prevalent (both theory and empiricism suggest they are; Jones et al. 1994, 1997, 2010, Hastings et al. 2007) then this may require empirical parameterization of functions relating engineer density and per capita engineering activity (Cuddington et al. 2009) to abiotic change; their relationships to food web members including the engineer (e.g. lagged demographic responses); tendencies for subsequent change in engineered abiotic states (e.g. environmental decay; Raynaud et al. 2013); and abiotic interaction terms (e.g. how engineer maintenance activity in response to one abiotic change then alters susceptibility to other abiotic factors). 
Fourth, we need to merge the three above approaches into an integrated general understanding of how engineering can alter food web structure and dynamics and vice versa. This could be achieved using models in the spirit of Goudard and Loreau (2008), Arditi et al. (2005), and Kéfi et al. (2012), along with necessary simplifications. We might, for example: 1) build theoretical trophic networks involving realistically large numbers of species (as has often been done, Thebault and Fontaine 2010) that include engineer effects on nodes and links to elaborate general theory about the interaction between engineering and trophic interactions that is comparable to current theories linking food web architecture to their properties (Eklöf et al. 2013); 2) build dynamic models that allow modelling and parameterization of interactions between a limited number of food web species and engineers for real ecological systems; 3) use already parameterized realistic food webs (Boit et al. 2012), to experimentally document engineer effects, including the findings in models to explore general impacts on food web stability and structure.

Combining the above approaches might provide general answers to the following kinds of questions: When does food web/engineer interactions result in new kinds of dynamics, such as alternate stable equilibria? When do engineers stabilize or destabilize biomass or species richness of food webs? What are the community wide consequences of being an engineer and prey, or engineer and predator? How does having a significant engineer in a food web alter the way the web will respond to external forcing when the engineer does or does not modify that forcing (e.g. climate change with and without engineered microclimates)? Should links between species and abiotic variables be treated in the same way as trophic links to calculate network metrics (e.g., connectance, modularity, nestedness)?

While our framework, models, and strategies for research in this area are deliberately general, we believe that investigators can readily make these operational in their systems, constraining their research to the aspects of engineering that are the most important and influential, and focussing on the most relevant spatial scales and time scales that can capture influential feedbacks.

Finally, while answering questions about reciprocal influences of engineering and food webs, such an approach also paves the way for comparing these kinds of interaction webs with other kinds of interaction webs (Olff et al. 2009, Bascompte 2010, Kéfi et al. 2012), bringing us back to the calls for broadening the scope of food web studies to include non-trophic interactions that we articulated in the introduction.

\section{Conclusion}

The recognition that organismal responses to the abiotic environment must include the fact that organisms can change the abiotic environment is central to ecosystem engineering theory, as is the recognition that such changes can affect those organisms, other organisms and ecological processes. Nevertheless, such realities have not been yet been substantively integrated into food web theory. We think that we can no longer sensibly treat food web and engineering dynamics as independent if we seek to understand either; and we certainly cannot do so if we seek to understand reality. In general, our understanding of how engineering shapes food webs and vice versa is perhaps more hampered by lack of knowledge about food web responses to abiotic change than knowledge about how ecosystem engineers can cause such change; and this is compounded by the fact that engineering and food web studies often insufficiently overlap in the same systems. Yet with appropriate studies and integrative models, we believe that conjunction is achievable, and that this can also then pave the way to a more general understanding of interaction webs in nature that takes into account the often complex interactions among and between biota and the abiotic environment.

Acknowledgements - This paper is a product of the Working Group 'Ecosystem Engineering and Scaling' convened at the Centre for Estuarine and Marine Ecology, Netherlands Inst. of Ecology (NIOO), Yerseke, the Netherlands (now the Royal Netherlands Institute for Sea Research) in April and June 2011, and continued thereafter. Workshops were organized by CGJ, TB and Peter Herman and financially supported by KNAW grant ISK/6445/ VPP to CGJ and the NIOO. We thank Joke van Houte, Anneke van der Endt, Elly van Hulsteijn, Christine van der Jagt, Laura Wisse and Jan Megans for logistical support of the workshops. CGJ thanks The Royal Netherlands Academy for Arts and Sciences and The Cary Inst. of Ecosystem Studies for financial support. JvB thanks the European Union THESEUS Project for financial support. We thank Robert D. Holt and Jeb Byers for valuable comments on the manuscript. This is a contribution to the program of the Cary Institute of Ecosystem Studies. The authors declare no conflict of interest.

\section{References}

Anderson, C. and Rosemond, A. 2010. Beaver invasion alters terrestrial subsidies to subantarctic stream food webs. - Hydrobiologia 652: 349-361.

Arditi, R. et al. 2005. Rheagogies: modelling non-trophic effects in food webs. - Ecol. Complex. 2: 249-258.

Badano, E. I. et al. 2006. Assessing impacts of ecosystem engineers on community organization: a general approach illustrated by effects of a high-Andean cushion plant. - Oikos 115: 369-385.

Barot, S. et al. 2007. Nutrient cycling efficiency explains the long-term effect of ecosystem engineers on primary production. - Funct. Ecol. 21: 1-10.

Bascompte, J. 2010. Structure and dynamics of ecological networks. - Science 329: 765-766.

Boit, A. et al. 2012. Mechanistic theory and modelling of complex food-web dynamics in Lake Constance. - Ecol. Lett. 15: 594-602.

Bos, A. R. et al. 2007. Ecosystem engineering by annual intertidal seagrass beds: sediment accretion and modification. - Estuar. Coast. Shelf S. 74: 344-348.

Brody, A. K. et al. 2010. Termites, vertebrate herbivores, and the fruiting success of Acacia drepanolobium. - Ecology 91: 399-407.

Brown, C. J. et al. 2010. Effects of climate-driven primary production change on marine food webs: implications for fisheries and conservation. - Global Change Biol. 16: 1194-1212.

Bruno, J. F. 2000. Facilitation of cobble beach plant communities through habitat modification by Spartina alterniflora. - Ecology 81: 1179-1192. 
Chase, J. M. 1996. Abiotic controls of trophic cascades in a simple grassland food chain. - Oikos 77: 495-506.

Cross, W. F. et al. 2006. Whole-system nutrient enrichment increase secondary production in a detritus-based ecosystem. - Ecology 87: 1556-1565.

Cuddington, K. et al. 2009. Ecosystem engineers: feedback and population dynamics. - Am. Nat. 173: 488-498.

Daborn, G. R. et al. 1993. An ecological cascade effect: migratory birds affect stability of intertidal sediments. - Limnol. Oceanogr. 38: 225-231.

Dangerfield, J. M. et al. 1998. The mound-building termite Macrotermes michaelseni as an ecosystem engineer. - J. Trop. Ecol. 14: 507-520.

Dayton, P. K. 1985. Ecology of kelp communities. - Annu. Rev. Ecol. Syst. 16: 215-245.

Eklöf, A. et al. 2013. The dimensionality of ecological networks. - Ecol. Lett. 16: 577-583.

Estes, J. A. and Palmisano, J. F. 1974. Sea otters: their role in structuring nearshore communities. - Science 185: 1058-1060.

Estes, J. A. et al. 1998. Killer whale predation on sea otters linking oceanic and nearshore ecosystems. - Science 282: 473-476.

Golubski, A. J. and Abrams, P. A. 2011. Modifying modifiers: what happens when interspecific interactions interact? - J. Anim. Ecol. 80: 1097-1108.

Goudard, A. and Loreau, M. 2008. Nontrophic interactions, biodiversity, and ecosystem functioning: an interaction web model. - Am. Nat. 171: 91-106.

Grabowski, J. H. and Powers, S. P. 2004. Habitat complexity mitigates trophic transfer on oyster reefs. - Mar. Ecol. Progr. Ser. 277: 291-295.

Gutiérrez, J. and Iribarne, O. 1999. Role of Holocene beds of the stout razor clam Tagelus plebeius in structuring present benthic communities. - Mar. Ecol. Progr. Ser. 185: 213-228.

Gutiérrez, J. L. and Jones, C. G. 2006. Physical ecosystem engineers as agents of biogeochemical heterogeneity. - BioScience 56: 227-236.

Gutiérrez, J. L. et al. 2003. Mollusks as ecosystem engineers: the role of shell production in aquatic habitats. - Oikos 101: 79-90.

Gutiérrez, J. L. et al. 2006. The contribution of crab burrow excavation to carbon availability in surficial salt-marsh sediments. - Ecosystems 9: 647-658.

Hansen, K. and Kristensen, E. 1997. Impact of macrofaunal recolonization on benthic metabolism and nutrient fluxes in a shallow marine sediment previously overgrown with macroalgal mats. - Est. Coast. Shelf Sci. 45: 613-628.

Hastings, A. et al. 2007. Ecosystem engineering in space and time. - Ecol. Lett. 10: 153-164.

Holt, J. A. and Lepage, M. 2000. Termites and soil properties. Termites: evolution, sociality, symbioses, ecology. Kluwer Academic Publishers, pp. 389-407.

Holt, R. D. and Barfield, M. 2013. Direct plant-predator interactions as determinants of food chain dynamics. - J. Theor. Biol. 339: 47-57.

Hopcraft, J. G. C. et al. 2005. Planning for success: Serengeti lions seek prey accessibility rather than abundance. - J. Anim. Ecol. 74: 559-566.

Hulot, F. D. et al. 2000. Functional diversity governs ecosystem response to nutrient enrichment. - Nature 405: 340-344.

Ings, T. C. et al. 2009. Ecological networks - beyond food webs. - J. Anim. Ecol. 78: 253-269.

Jones, C. G. 2012. Ecosystem engineers and geomorphological signatures in landscapes. - Geomorphology 157/158: 75-87.

Jones, C. G. and Gutierrez, J. L. 2007. On the purpose, meaning, and usage of the physical ecosystem engineering concept. - In:
Cuddington, K. et al. (eds), Ecosystem engineers. Academic Press/Elsevier, p. 405.

Jones, C. G. et al. 1994. Organisms as ecosystem engineers. - Oikos 69: 373-386.

Jones, C. G. et al. 1997. Positive and negative effects of organisms as physical ecosystem engineers. - Ecology 78: 1946-1957.

Jones, C. G. et al. 2010. A framework for understanding physical ecosystem engineering by organisms. - Oikos 119: 1862-1869.

Jonkman, J. C. M. 1980. The external and internal structure and growth of nests of the leaf-cutting ant Atta vollenweideri Forel, 1893 (Hym.: Formicidae). - Z. Angew. Entomol. 89: 217-246.

Kéfi, S. et al. 2012. More than a meal... integrating nonfeeding interactions into food webs. - Ecol. Lett. 15: 291-300.

Kitching, R. L. 1971. An ecological study of water-filled treeholes and their position in the woodland ecosystem. - J. Anim. Ecol. 40: 281-302.

Knight, D. et al. 1992. The role of earthworms in managed, permanent pastures in Devon, England. - Soil Biol. Biochem. 24: 1511-1517.

Lavelle, P. et al. 2006. Soil invertebrates and ecosystem services. - Eur. J. Soil Biol. 42: S3-S15.

Mayfield, M. M. et al. 2009. Traits, habitats, and clades: identifying traits of potential importance to environmental filtering. - Am. Nat. 174: E1-E22.

McCann, K. and Yodzis, P. 1995. Bifurcation structure of a three-species food-chain model. - Theor. Popul. Biol. 48: 93-125.

McCann, K. and Hastings, A. 1997. Re-evaluating the omnivory-stability relationship in food webs. - Proc. R. Soc. B 264: 1249-1254.

McCann, K. et al. 1998a. Weak trophic interactions and the balance of nature. - Nature 395: 794-798.

McCann, K. S. et al. 1998b. Trophic cascades and trophic trickles in pelagic food webs. - Proc. R. Soc. B 265: 205-209.

Medlock, J. M. and Snow, K. R. 2008. Natural predators and parasites of British mosquitoes - a review. - Eur. Mosquito Bull. 25: 1-11.

Micheli, F. 1999. Eutrophication, fisheries and consumer-resource dynamics in marine pelagic ecosystems. - Science 285: 1396-1398.

Montoya, J. M. et al. 2006. Ecological networks and their fragility. - Nature 442: 259-264.

Moore, J. C. et al. 1993. Influence of productivity on the stability of real and model-ecosystems. - Science 261: 906-908.

Mougi, A. and Kondoh, M. 2012. Diversity of interaction types and ecological community stability. - Science 337: 349-351.

Nagelkerken, I. et al. 2000. Importance of mangroves, seagrass beds and the shallow coral reef as a nursery for important coral reef fishes, using a visual census technique. - Est. Coast. Shelf Sci. 51: 31-44.

Olff, H. et al. 2009. Parallel ecological networks in ecosystems. - Phil. Trans. R. Soc B 364: 1755-1779.

Otto, S. B. et al. 2007. Allometric degree distributions facilitate food-web stability. - Nature 450: 1226-1229.

Pace, M. L. et al. 1999. Trophic cascades revealed in diverse ecosystems. - Trends Ecol. Evol. 14: 483-488.

Parmelee, R. W. et al. 1998. Earthworms and nutrient cycling processes: integrating across the ecological hierarchy. - In: Edwards, C. A. (ed.), Earthworm ecology. St. Lucie Press, pp. 123-143.

Pearson, D. E. 2010. Trait- and density-mediated indirect interactions initiated by an exotic invasive plant autogenic ecosystem engineer. - Am. Nat. 176: 394-403. 
Petchey, O. L. et al. 2010. Predicting the effects of temperature on food web connectance. - Phil. Trans. R. Soc B 365: 2081-2091.

Pimm, S. L. 1982. Food webs. - Chapman and Hall.

Pintor, L. M. and Soluk, D. A. 2006. Evaluating the nonconsumptive, positive effects of a predator in the persistence of an endangered species. - Biol. Conserv 130: 584-591.

Prugh, L. R. and Brashares, J. S. 2012. Partitioning the effects of an ecosystem engineer: kangaroo rats control community structure via multiple pathways. - J. Anim. Ecol. 81: 667-678.

Radke, R. J. and Gaupisch, A. 2005. Effects of phytoplanktoninduced turbidity on predation success of piscivorous Eurasian perch (Perca fluviatilis): possible implications for fish community structure in lakes. - Naturwissenschaften 92: 91-94.

Raynaud, X. et al. 2013. Ecosystem engineering, environmental decay and environmental states of landscapes. - Oikos 122: 591-600.

Rooney, N. et al. 2006. Structural asymmetry and the stability of diverse food webs. - Nature 442: 265-269.

Rosenzweig, M. L. 1971. Paradox of enrichment: destabilization of exploitation ecosystems in ecological time. - Science 171: 385-387.

Rosenzweig, M. L. 1973. Exploitation in 3 trophic levels. - Am. Nat. 107: 275-294.

Sanders, D. and van Veen, F. J. F. 2011. Ecosystem engineering and predation: the multi-trophic impact of two ant species. - J. Anim. Ecol. 80: 569-576.

Schmitz, O. J. 2010. Resolving ecosystem complexity. - Pinceton Univ. Press.
Shachak, M. et al. 2008. Woody species as landscape modulators and their effect on biodiversity patterns. - BioScience 58: 209-221.

Solé, R. V. and Montoya, M. 2001. Complexity and fragility in ecological networks. - Proc. R. Soc. B 268: 2039-2045.

Strayer, D. L. et al. 1999. Transformation of freshwater ecosystems by bivalves. - BioScience 49: 19-27.

Thebault, E. and Fontaine, C. 2010. Stability of ecological communities and the architecture of mutualistic and trophic networks. - Science 329: 853-856.

Thomas, J. A. et al. 2009. Successful conservation of a threatened maculinea butterfly. - Science 325: 80-83.

Tilman, D. et al. 2001. Forecasting agriculturally driven global environmental change. - Science 292: 281-284.

Tribollet, A. and Golubic, S. 2011. Reef bioerosion: agents and processes. - In: Dubinsky, Z. and Stambler, N. (eds), Coral reefs: an ecosystem in transition. Springer, pp. 435-449.

van Veen, F. J. F. et al. 2008. Food web structure of three guilds of natural enemies: predators, parasitoids and pathogens of aphids. - J. Anim. Ecol. 77: 191-200.

Vitousek, P. M. et al. 1997. Human alteration of the global nitrogen cycle: sources and consequences. - Ecol. Appl. 7: 737-750.

Woodward, G. et al. 2010. Sentinel systems on the razor's edge: effects of warming on Arctic geothermal stream ecosystems. - Global Change Biol. 16: 1979-1991.

Wright, J. P. and Jones, C. G. 2006. The concept of organisms as ecosystem engineers ten years on: progress, limitations and challenges. - Bioscience 56: 203-209.

Wright, J. P. et al. 2006. Predictability of ecosystem engineering effects on species richness across environmental variability and spatial scales. - J. Ecol. 94: 815-824. 